\title{
SPACE-TIME ESTIMATES IN THE BESOV SPACES AND THE NAVIER-STOKES EQUATIONS*
}

\author{
QIONGLEI CHEN ${ }^{\dagger}$ AND ZHIFEI ZHANG‡
}

\begin{abstract}
In this paper, we establish the space-time estimates in the Besov spaces of the solution to the Navier-Stokes equations in $\mathbf{R}^{n}, n \geq 3$. As an application, we improve some known results about the regularity criterion of weak solutions and the blow-up criterion of smooth solutions. Our main tools are the frequency localization and the Littlewood-Paley decomposition.
\end{abstract}

Key words. Navier-Stokes equations, Besov space, Regularity criterion, Littlewood-Paley decomposition

AMS subject classifications. 35Q30, 35D10, 76D03, 76D05

1. Introduction. In this paper, we are concerned with the regularity of weak solutions and the blow-up phenomena of smooth solutions to the Navier-Stokes equations in $\mathbf{R}^{n}, n \geq 3$ :

$$
\text { (NS) }\left\{\begin{array}{lll}
u_{t}-\Delta u+u \cdot \nabla u+\nabla \pi=0, & \text { in } \mathbf{R}^{n} \times(0, T), \\
\nabla \cdot u=0, & \text { in } \mathbf{R}^{n} \times(0, T), \\
u(0)=u_{0}(x), & \text { in } \mathbf{R}^{n},
\end{array}\right.
$$

where $u=\left(u^{1}(t, x), \cdots, u^{n}(t, x)\right)$ and $\pi=\pi(t, x)$ denote the unknown velocity vector and the unknown scalar pressure of the fluid at the point $(t, x) \in(0, T) \times \mathbf{R}^{n}$, respectively, while $u_{0}=\left(u_{0}^{1}(x), \cdots, u_{0}^{n}(x)\right)$ is a given initial velocity vector satisfying $\nabla \cdot u_{0}=0$. Here we use the notation:

$$
u \cdot \nabla v=\sum_{i=1}^{n} u^{i} \frac{\partial v}{\partial x_{i}}, \quad \nabla \cdot u=\sum_{i=1}^{n} \frac{\partial u^{i}}{\partial x_{i}}
$$

for vector functions $u, v$.

For given $u_{0} \in L^{2}\left(\mathbf{R}^{n}\right)$ with $\nabla \cdot u_{0}=0$, J. Leray and E. Hopf [17, 20](see also $[21,30])$ constructed a global weak solution $u(t, x) \in L^{\infty}\left(0, \infty ; L^{2}\left(\mathbf{R}^{n}\right)\right) \cap$ $L^{2}\left(0, \infty ; H^{1}\left(\mathbf{R}^{n}\right)\right)$. It is well known that the weak solution is unique and regular in two spatial dimensions[11,30]. In dimensions $n \geq 3$, however, the question of regularity of weak solutions has remained open in spite of much effort of mathematicians. There are two ways to develop the regularity theory for the Navier-Stokes equations. One is to give a regularity criterion on weak solutions and the other is to study a better partial regularity of weak solutions which are known to exist. For the latter, L. Caffarelli, R. Kohn, L. Nirenberg[4] proved that the one dimensional Hausdorff measure of the singular set is zero, which is the best result at present. J. Serrin[24, 25] is the pioneer in the former research, later on, Fabes, Jones and Riviere[13], Sohr[26], Giga[16], Struwe[27] and Takahashi[28] extended and improved Serrin's regularity criterion. In order to state their result, we first recall the definition of weak solutions to the Navier-Stokes equations.

Definition 1.1. Let $u_{0} \in L^{2}\left(\mathbf{R}^{n}\right)$ with $\nabla \cdot u_{0}=0$. The function $u(t, x)$ will be called a weak solution of $(N S)$ on $(0, T)$ if u satisfies the following properties:

\footnotetext{
${ }^{*}$ Received April 6, 2005; accepted for publication August 25, 2006.

$\dagger$ Institute of Applied Physics and Computational Mathematics, Beijing 100088, China (qleichen @yahoo.com.cn).

${ }^{\ddagger}$ School of Mathematics, Peking University, Beijing 100871, China (zfzhang@math.pku.edu.cn).
} 
(1) $u \in L^{\infty}\left(0, T ; L^{2}\left(\mathbf{R}^{n}\right)\right) \cap L^{2}\left(0, T ; H^{1}\left(\mathbf{R}^{n}\right)\right)$;

(2) $u_{t}-\Delta u+u \cdot \nabla u+\nabla \pi=0$ in $\mathcal{D}^{\prime}\left(\mathbf{R}^{n} \times(0, T)\right)$;

(3) $\nabla \cdot u=0$ in $\mathcal{D}^{\prime}\left(\mathbf{R}^{n} \times(0, T)\right)$;

(4) (The Energy Inequality)

$$
\|u(t)\|_{2}^{2}+2 \int_{0}^{t}\|\nabla u(\tau)\|_{2}^{2} d \tau \leq\left\|u_{0}\right\|_{2}^{2}, \quad 0<t<T
$$

Their result now reads:

Proposition 1.1. Suppose that $u(t, x)$ is a weak solution of $(N S)$ on $(0, T)$, and satisfies

$$
\int_{0}^{T}\|u(t)\|_{r}^{q} d t<\infty \quad \text { for } \quad \frac{2}{q}+\frac{n}{r}=1 \quad n<r \leq \infty .
$$

Then $u$ is regular on $(0, T]$, that is $u \in C^{\infty}\left((0, T] \times \mathbf{R}^{n}\right)$.

Instead of the solution itself, H. Beirão da Veiga[1] proved the following regularity criterion in terms of the gradient of the solution.

Proposition 1.2. Suppose that $u(t, x)$ is a weak solution of $(N S)$ on $(0, T)$, and satisfies

$$
\int_{0}^{T}\|\nabla u(t)\|_{r}^{q} d t<\infty \quad \text { for } \quad \frac{2}{q}+\frac{n}{r}=2 \quad \frac{n}{2}<r \leq \infty .
$$

Then the weak solution $u$ is regular in $(0, T]$.

REMARK 1.1. Since the gradient of velocity can be described by the vorticity through the singular integral operator, for example, for $n=3, \nabla u=$ $(-\triangle)^{-1} \nabla$ rotrotu, Proposition 1.2 also states a condition for the vorticity rotu if $r<\infty$.

Recently H. Kozono, T. Ogawa, and Y. Taniuchi [19] refined the condition (1.3) to

$$
\int_{0}^{T}\|\operatorname{rot} u(t)\|_{\dot{B}_{r}^{0, \infty}}^{q} d t<\infty \quad \text { for } \quad \frac{2}{q}+\frac{n}{r}=2 \quad n \leq r \leq \infty .
$$

Here and thereafter, $\dot{B}_{p}^{s, q}$ stands for the homogenous Besov space, see Definition 1.2. Their proof is based on the logarithmic Sobolev inequality in the Besov spaces, and the authors established a priori estimate with a double exponential growth, that is

$$
\|u(t)\|_{H^{s}} \leq\left\|u_{0}\right\|_{H^{s}} \exp \left\{\exp \left(C \int_{0}^{t}\left(1+\|\operatorname{rot} u(\tau)\|_{\dot{B}_{r}^{0, \infty}}\right)^{q} d \tau\right)\right\},
$$

where $s>\frac{n}{2}+1, \frac{2}{q}+\frac{n}{r}=2, n \leq r \leq \infty$.

On the other hand, for $u_{0} \in H^{s}\left(\mathbf{R}^{n}\right), s \geq \frac{n}{2}-1$, H. Fujita and T. Kato[14] proved that there exists $T=T\left(\left\|u_{0}\right\|_{H^{s}}\right)$ and a unique strong solution $u(t)$ of (NS) on $[0, T)$ in the class

$$
(C L)_{s} \quad u \in C\left([0, T) ; H^{s}\right) \cap C^{1}\left((0, T) ; H^{s}\right) \cap C\left((0, T) ; H^{s+2}\right) .
$$


It is a natural question whether $u(t)$ loses its regularity at $t=T$. Y. Giga[16] showed that if the above strong solution $u(t)$ satisfies the condition

$$
\int_{0}^{T}\|u(t)\|_{r}^{q} d t<\infty \quad \text { for } \quad \frac{2}{q}+\frac{n}{r}=1, \quad n<r \leq \infty,
$$

then $u$ can be continued to the solution in the class $(C L)_{s}$ beyond $t=T$. Recently, in the endpoint case(i.e. $r=\infty$ ), based on the bilinear estimates in the BMO space for the nonlinear term $u \cdot \nabla u, \mathrm{H}$. Kozono and Y. Tanuchi[18] showed if

$$
\int_{0}^{T}\|u(t)\|_{B M O}^{2} d t<\infty
$$

then the strong solution $u(t)$ in the class $(C L)_{s}$ on $[0, T)$ can be continued to the one with the same regularity on for some $T^{\prime}>T$. Moreover, the authors obtained the single exponential estimate of the solution. However, using the logarithmic Sobolev inequality, the $L^{\infty}$-theory can only give a priori estimate with the double exponential growth.

The main purpose of this paper is to improve some known results about the regularity criterion of weak solutions and the blow-up criterion of smooth solutions. Instead of the logarithmic Sobolev inequality, our main tools are the frequency localization and the Littlewood-Paley trichotomy decomposition, which is a basic way to analysis bilinear expressions. These techniques have been widely used in the study of the fluid dynamics equations, see $[6,7,12,15,22,32]$ and reference therein.

Before stating main results, let's introduce some notations and definitions. Let $\mathcal{S}\left(\mathbf{R}^{n}\right)$ be the Schwartz class of rapidly decreasing functions. Given $f \in \mathcal{S}\left(\mathbf{R}^{n}\right)$, its Fourier transform $\hat{f}$ is defined by

$$
\hat{f}(\xi)=\int_{\mathbf{R}^{n}} e^{-i x \cdot \xi} f(x) d x .
$$

Let us choose a nonnegative radial function $\phi \in \mathcal{S}\left(\mathbf{R}^{n}\right)$ such that

$$
0 \leq \hat{\phi}(\xi) \leq 1, \quad \hat{\phi}(\xi)=\left\{\begin{array}{lll}
1, & \text { if } \quad|\xi| \leq 1 \\
0, & \text { if } \quad|\xi| \geq 2
\end{array}\right.
$$

We also introduce another function given by

$$
\psi(x)=\phi(x)-2^{-n} \phi(x / 2) .
$$

Set

$$
\phi_{j}(x)=2^{j n} \phi\left(2^{j} x\right), \quad \psi_{j}(x)=2^{j n} \psi\left(2^{j} x\right), \quad j \in \mathbb{Z} .
$$

Then the Littlewood-Paley projection operators $P_{\leq j}$ and $P_{j}$ are respectively defined by

$$
P_{\leq j} f=\phi_{j} * f, \quad P_{j} f=\psi_{j} * f, \quad j \in \mathbb{Z} .
$$

Informally, $P_{j}$ is a frequency projection to the annulus $\left\{|\xi| \sim 2^{j}\right\}$, while $P_{\leq j}$ is a frequency projection to the ball $\left\{|\xi| \lesssim 2^{j}\right\}$. We shall sometimes write $P_{<j}$ for $P_{\leq j-1}$. We also define more general projections by

$$
P_{k_{1} \leq . \leq k_{2}} f=\sum_{j=k_{1}}^{k_{2}} P_{j} f .
$$


Definition 1.2. Let $s \in \mathbf{R}, p, q \in[1, \infty]$, the homogeneous Besov space $\dot{B}_{p}^{s, q}\left(\mathbf{R}^{n}\right)$ is defined by

$$
\dot{B}_{p}^{s, q}\left(\mathbf{R}^{n}\right):=\left\{f \in \mathcal{Z}^{\prime}\left(\mathbf{R}^{n}\right) ;\|f\|_{\dot{B}_{p}^{s, q}}<+\infty\right\}
$$

Here

$$
\|f\|_{\dot{B}_{p}^{s, q}}=\left\{\begin{array}{l}
\left(\sum_{j=-\infty}^{+\infty} 2^{s j q}\left\|P_{j} f\right\|_{p}^{q}\right)^{\frac{1}{q}}, \quad \text { if } q<+\infty \\
\sup _{j \in \mathbb{Z}} 2^{s j}\left\|P_{j} f\right\|_{p}, \quad \text { if } q=+\infty
\end{array}\right.
$$

and $\mathcal{Z}^{\prime}\left(\mathbf{R}^{n}\right)$ denotes the dual space of $\mathcal{Z}\left(\mathbf{R}^{n}\right)=\left\{f \in \mathcal{S}\left(\mathbf{R}^{n}\right) ; D^{\alpha} \hat{f}(0)=0 ; \forall \alpha \in\right.$ $\mathbf{N}^{n}$ multi-index $\}$ and can be identified by the quotient space of $\mathcal{S}^{\prime} / \mathcal{P}$ with the polynomials space $\mathcal{P}$. We refer to $[2,31]$ for more details. by

We will also need a mixed space-time space $\widetilde{L}^{r}\left(a, b ; \dot{B}_{p}^{s, q}\right)$, whose norm is defined

$$
\|u\|_{\tilde{L}^{r}\left(a, b ; \dot{B}_{p}^{s, q}\right)}:=\left\{\begin{array}{l}
\left(\sum_{j=-\infty}^{+\infty} 2^{s j q}\left\|P_{j} f\right\|_{L^{r}\left(a, b ; L_{x}^{p}\right)}^{q}\right)^{\frac{1}{q}}, \quad \text { if } q<+\infty, \\
\sup _{j \in \mathbb{Z}} 2^{s j}\left\|P_{j} f\right\|_{L^{r}\left(a, b ; L_{x}^{p}\right)}, \quad \text { if } q=+\infty .
\end{array}\right.
$$

For the convenience, we sometimes use $\widetilde{L}_{T}^{r} \dot{B}_{p}^{s, q}$ to denote $\widetilde{L}^{r}\left(0, T ; \dot{B}_{p}^{s, q}\right)$. For the introduction of this type of space, we refer to [8].

Now we state the space-time estimates in the Besov spaces of the solution to the Navier-Stokes equations, which might be of independent interest.

TheOREM 1.1. Let $p, q, r, \sigma, \gamma, s$ satisfy

$$
\begin{aligned}
& p \in[2, \infty), q \in[1, \infty),(r, \sigma) \in[1, \infty], \gamma \in(-1,1] \\
& \frac{2}{q}+\frac{n}{r}=1+\gamma, \frac{n}{r}-\gamma+1+2 s>0, \text { and }(r, \gamma) \neq(\infty, 1) .
\end{aligned}
$$

Assume that $u$ is a smooth solution to the Navier-Stokes equations on $[0, T)$ with the initial data $u_{0} \in \dot{B}_{p}^{s, \sigma}$. Then there holds

$$
\|u\|_{{\widetilde{L_{T}}}^{\infty} \dot{B}_{p}^{s, \sigma}}^{2}+\|u\|_{{\widetilde{L_{T}}}^{2} \dot{B}_{p}^{s+1, \sigma}}^{2} \leq C\left\|u_{0}\right\|_{\dot{B}_{p}^{s, \sigma}}^{2}+C\|u\|_{{\widetilde{L_{T}}}^{q} \dot{B}_{r}^{\gamma, \infty}}^{q}\|u\|_{\widetilde{L}_{T}{ }^{\infty} \dot{B}_{p}^{s, \sigma}}^{2}
$$

REMARK 1.2. If the solution $u(t)$ satisfies

$$
A \equiv \int_{0}^{T}\|u(t)\|_{\dot{B}_{r}^{\gamma}, \infty}^{q} d t<+\infty
$$

i.e., $u(t) \in L^{q}\left(0, T ; \dot{B}_{r}^{\gamma, \infty}\right)$, then we can deduce the following single exponential estimate

$$
\|u\|_{\widetilde{L}_{T}{ }^{\infty} \dot{B}_{p}^{s, \sigma}}^{2}+\|u\|_{{\widetilde{L_{T}}}^{2} \dot{B}_{p}^{s+1, \sigma}}^{2} \leq C\left\|u_{0}\right\|_{\dot{B}_{p}^{s, \sigma}}^{2} \exp \left(C^{\prime} A\right) .
$$

In fact, take $\varepsilon_{0}>0$ such that $C \varepsilon_{0} \leq \frac{1}{2}$, where $C$ is the constant appearing in Theorem 1.4. If $A \leq \varepsilon_{0}$, then from Theorem 1.4 it follows that

$$
\|u\|_{\widetilde{L}_{T}^{\infty} \dot{B}_{p}^{s, \sigma}}^{2}+\|u\|_{{\widetilde{L_{T}}}^{2} \dot{B}_{p}^{s+1, \sigma}}^{2} \leq 2 C\left\|u_{0}\right\|_{\dot{B}_{p}^{s, \sigma}}^{2},
$$


since $\|u\|_{\tilde{L}^{q}\left(0, T ; B_{r}^{\gamma, \infty}\right)} \leq\|u\|_{L^{q}\left(0, T ; B_{r}^{\gamma, \infty}\right)}$. Otherwise, we choose $0=T_{0}<T_{1}<\cdots<$ $T_{N}=T_{N+1}=T$ so that

$$
\int_{T_{i}}^{T_{i+1}}\|u(t)\|_{\dot{B}_{r}^{\gamma, \infty}}^{q} d t=\varepsilon_{0}, \quad i=0, \cdots N-1, \quad \int_{T_{N}}^{T_{N+1}}\|u(t)\|_{\dot{B}_{r}^{\gamma, \infty}}^{q} d t \leq \varepsilon_{0} .
$$

Then

$$
\int_{0}^{T}\|u(t)\|_{\dot{B}_{r}^{\gamma, \infty}}^{q} d t=\sum_{i=0}^{N-1} \int_{T_{i}}^{T_{i+1}}\|u(t)\|_{\dot{B}_{r}^{\gamma, \infty}}^{q} d t \geq N \varepsilon_{0} .
$$

Repeating the argument that leads to (1.7) $N$ times yields

$$
\|u\|_{{\widetilde{L_{T}}}^{\infty} \dot{B}_{p}^{s, \sigma}}^{2}+\|u\|_{{\widetilde{L_{T}}}^{2} \dot{B}_{p}^{s+1, \sigma}}^{2} \leq 2^{N} C\left\|u_{0}\right\|_{\dot{B}_{p}^{s, \sigma}}^{2}, \quad 0 \leq T \leq T_{N} .
$$

Once we use (1.8) we get (1.6) with $C^{\prime}=\varepsilon_{0}^{-1} \log 2$.

If we take $p=\sigma=2$ in (1.6), we get

$$
\|u\|_{L_{T}^{\infty} H^{s}}^{2}+\|u\|_{L_{T}^{2} H^{s+1}}^{2} \leq C\left\|u_{0}\right\|_{H^{s}}^{2} \exp \left(C^{\prime} A\right),
$$

which is sharper than the estimate (1.5).

REMARK 1.3. By using the analytic semigroup approach, Chen and Xin[10] derived similar estimates in the critical homogenous spaces and established the global existence of small regular solutions.

REMARK 1.4. Based on the characterization of Besov spaces in terms of the heat semigroup and Kato's method, Cannone [5] proved the global well-posedness of the Navier-Stokes equations with small data in the critical Besov spaces. If we apply Theorem 1.1 with $p=r, q=2, s=\frac{n}{r}-1$, we can provide a different proof of his result.

As an application of Theorem 1.1, we obtain the blow-up criterion of smooth solutions to the Navier-Stokes equations.

TheOrem 1.2. Let $u_{0} \in H^{s}\left(\mathbf{R}^{n}\right), s>\frac{n}{2}-1$. Assume that $u$ is a strong solution of $(N S)$ in the class $(C L)_{s}(0, T)$. If there exists a small $\varepsilon_{0}>0$ such that

$$
\lim _{\varepsilon \rightarrow 0} \sup _{j} 2^{j \gamma q} \int_{T-\varepsilon}^{T}\left\|P_{j} u(t)\right\|_{r}^{q} d t \leq \varepsilon_{0}
$$

where $q, r, \gamma$ satisfy $\frac{2}{q}+\frac{n}{r}=1+\gamma, \frac{n}{1+\gamma}<r \leq \infty,-1<\gamma \leq 1$, and $(r, \gamma) \neq(\infty, 1)$. Then $u$ can be continued to the strong solution in the class $(C L)_{s}\left(0, T^{\prime}\right)$ for some $T^{\prime}>T$.

REMARK 1.5. Notice that if $u(t) \in L^{q}\left(0, T ; L^{r}\right), \frac{2}{q}+\frac{n}{r}=1, n<r \leq \infty$, then

$$
\lim _{\varepsilon \rightarrow 0} \sup _{j} \int_{T-\varepsilon}^{T}\left\|P_{j} u(t)\right\|_{r}^{q} d t \leq \lim _{\varepsilon \rightarrow 0} \int_{T-\varepsilon}^{T}\|u(t)\|_{r}^{q} d t=0,
$$

therefore our result improves Giga's result[16]. 
REMARK 1.6. For the incompressible Euler equation, Planchon [23] proved a blow-up criterion of smooth solutions in the same kind of mixed Besov space. Therefore our result can be regarded as an extension of his result.

Next we apply Theorem 1.1 to the regularity criterion of weak solutions to the Navier-Stokes equations.

THEOREM 1.3. Let $q, r, \gamma$ be as in Theorem 1.2. Assume that $u(t)$ is a weak solution to the Navier-Stokes equations in $(0, T)$ with data $u_{0} \in H^{s}, s>\frac{n-2}{2}$. If there exists a small $\varepsilon_{0}$ such that for any $t \in(0, T)$, u satisfies

$$
\lim _{\varepsilon \rightarrow 0} \sup _{j} 2^{j \gamma q} \int_{t-\varepsilon}^{t}\left\|P_{j} u(\tau)\right\|_{r}^{q} d \tau \leq \varepsilon_{0}
$$

then $u$ is regular in $(0, T]$.

REMARK 1.7. If $u(t) \in L^{q}\left(0, T ; \dot{B}_{r}^{1, \infty}\right), q<+\infty$, then for $t \in(0, T)$

$$
\lim _{\varepsilon \rightarrow 0} \sup _{j} 2^{j q} \int_{t-\varepsilon}^{t}\left\|P_{j} u(t)\right\|_{r}^{q} d t \leq \lim _{\varepsilon \rightarrow 0} \int_{t-\varepsilon}^{t}\|u(t)\|_{\dot{B}_{r}^{1, \infty}}^{q} d t=0,
$$

thus, for $\frac{2}{q}+\frac{n}{r}=2$ with $\frac{n}{2}<r<\infty$, our result is an improvement of the result given by H. Kozono, T. Ogawa, and Y. Taniuchi [19].

REMARK 1.8. The known results of regularity criterion need to restrict $\gamma$ to be non-negative, while our result allows $\gamma$ to be negative. It seems to be impossible to obtain this result by using the logarithmic Sobolev inequality as in [19].

2. Littlewood-Paley decomposition. Let's firstly introduce the LittlewoodPaly decomposition. Notice that that $P_{j}=P_{\leq j}-P_{<j}$. Also, if $f$ is an $L^{2}$ function then $P_{\leq j} f \rightarrow 0$ in $L^{2}$ as $j \rightarrow-\infty$ and $P_{\leq j} f \rightarrow f$ in $L^{2}$ as $j \rightarrow+\infty$ (this is an easy consequence of Parseval's theorem). By telescoping the series, we thus have the homogeneous Littlewood-Paley decompositin

$$
f=\sum_{j=-\infty}^{+\infty} P_{j} f
$$

for all $f \in L^{2}$, where the summation is in the $L^{2}$ sense.

In order to analyze bilinear expressions such as the pointwise product $(f, g) \mapsto f g$, we introduce the Littlewood-Paley trichotomy decomposition[29], which is a modified version of Bony's paraproduct[3]. More precisely, one is interested in understanding the quantity $P_{k}(f g)$-the $k^{t h}$ frequency component. One can split $f, g$ into LittlewoodPaley pieces too, and write

$$
P_{k}(f g)=\sum_{k^{\prime}, k^{\prime \prime} \in \mathbb{Z}} P_{k}\left(P_{k^{\prime}} f P_{k^{\prime \prime}} g\right)
$$

Recall that $P_{k^{\prime}} f$ has Fourier support in $2^{k^{\prime}-1} \leq|\xi| \leq 2^{k^{\prime}+1}$ and $P_{k^{\prime \prime}}$ has Fourier support in $2^{k^{\prime \prime}-1} \leq|\xi| \leq 2^{k^{\prime \prime}+1}$, so that $\left(P_{k^{\prime}} f\right)\left(P_{k^{\prime \prime}} g\right)$ has Fourier support in the sum of these two annuli. This sum needs to intersect the annulus $2^{k-1} \leq|\xi| \leq 2^{k+1}$ in order for the summand to be non-zero. This has the following consequences. If 
$k^{\prime}<k-5$, then the summand vanishes unless $k-3 \leq k^{\prime \prime} \leq k+3$. Similarly, if $k^{\prime \prime}<k-5$, then the summand vanishes unless $k-3 \leq k^{\prime} \leq k+3$. If $k^{\prime}>k+5$, then the summand vanishes unless $k^{\prime}-3 \leq k^{\prime \prime} \leq k^{\prime}+3$. Putting this all together, we get

$$
\begin{aligned}
& P_{k}(f g)=P_{k}\left\{P_{<k-5} f P_{k-3 \leq-\leq k+3} g+P_{k-3 \leq \cdot \leq k+3} f P_{<k-5} g\right. \\
& \left.\quad+P_{k-5 \leq \cdot \leq k+5} f P_{k-5 \leq \cdot \leq k+5} g+\sum_{k^{\prime}, k^{\prime \prime}>k+5 ;\left|k^{\prime}-k^{\prime \prime}\right| \leq 3} P_{k^{\prime}} f P_{k^{\prime \prime}} g\right\} .
\end{aligned}
$$

In other words, there are four distinct interactions of $f$ and $g$ which give a non-zero contribution to $P_{k}(f g)$ :

- Low-high interactions: $f$ has frequency $\ll 2^{k}, g$ has frequency $\sim 2^{k}$.

- High-low interactions: $f$ has frequency $\sim 2^{k}, g$ has frequency $\ll 2^{k}$.

- Low-low interactions: $f$ and $g$ have frequency $\sim 2^{k}$.

- High-high interactions: $f$ and $g$ have frequency $\sim 2^{k^{\prime}}$ for some $2^{k^{\prime}} \gg 2^{k}$.

The equality (2.2) is called the Littlewood-Paley trichotomy decomposition, which plays an important role in the proof of Theorem 1.1.

Next we prove some lemmas that will be used in the proof of Theorem 1.1.

Lemma 2.1. Assume that $f \in L^{p}\left(\mathbf{R}^{n}\right)$, and supp $\hat{f} \subset\left\{\xi \in \mathbf{R}^{n} ;|\xi| \sim 2^{j}\right\}, j \in \mathbb{Z}$. Then there exists a constant $C$ independent of $f, j$ such that

$$
\begin{gathered}
\|f\|_{p} \leq C 2^{-j|\alpha|} \sup _{|\beta|=|\alpha|}\left\|D^{\beta} f\right\|_{p}, \quad 1 \leq p \leq \infty . \\
\left\|D^{\alpha} f\right\|_{q} \leq C 2^{j n\left(\frac{1}{p}-\frac{1}{q}\right)+j|\alpha|}\|f\|_{p}, \quad 1 \leq p \leq q \leq \infty,
\end{gathered}
$$

for any $\alpha \in \mathbf{N}_{0}^{n}$.

For a proof, we see [9].

Lemma 2.2. Let $j \in \mathbb{Z}, 2 \leq q<\infty, f \in \mathcal{S}\left(\mathbf{R}^{n}\right)$ and supp $\hat{f} \subset\left\{\xi \in \mathbf{R}^{n} ;|\xi| \sim 2^{j}\right\}$. Then we have the following inequality:

$$
\int_{\mathbf{R}^{n}}|f|^{q} d x \leq C 2^{-2 j} \int_{\mathbf{R}^{n}}|\nabla f|^{2}|f|^{q-2} d x .
$$

Here $C$ is a constant depending only on $q, n$.

Proof. Let $\left(\theta_{i}\right)_{1 \leq i \leq n}$ be a sequence of infinitely differential function on $\Sigma^{n-1}$ satisfying the following properties:

$$
\sum_{i=1}^{n} \theta_{i}(\xi)=1, \quad \operatorname{supp} \theta_{i} \subset\left\{\xi \in \Sigma^{n-1} ; \frac{1}{2 \sqrt{n}} \leq\left|\xi_{i}\right| \leq 1\right\} .
$$

We decompose

$$
f(x)=\sum_{i=1}^{n}\left(\theta_{i}\left(\frac{\xi}{|\xi|}\right) \hat{f}(\xi)\right)^{\vee}(x)=\sum_{i=1}^{n} f_{i}(x) .
$$

Thus the $L^{q}$-norm of $f$ can be written as

$$
\int_{\mathbf{R}^{n}}|f|^{q} d x=\int_{\mathbf{R}^{n}} f f|f|^{q-2} d x=\sum_{i=1}^{n} \int_{\mathbf{R}^{n}} f_{i} f|f|^{q-2} d x .
$$


For each $i \in\{1, \cdots, n\}$, we take a function $F_{i}$ such that $\hat{F}_{i}=\frac{1}{-\mathrm{i} \xi_{i}} \hat{f}_{i}(\xi)$, that is, $f_{i}(x)=-\partial_{x_{i}} F_{i}(x)$. Then we have

$$
\int_{\mathbf{R}^{n}}|f|^{q} d x=\sum_{i=1}^{n} \int_{\mathbf{R}^{n}}-\partial_{x_{i}} F_{i}(x) f|f|^{q-2} d x=\sum_{i=1}^{n} \int_{\mathbf{R}^{n}} F_{i} \partial_{x_{i}}\left(f|f|^{q-2}\right) d x,
$$

where we have used the integration by parts in the last equality. It follows from the above equality and the Hölder inequality that

$$
\int_{\mathbf{R}^{n}}|f|^{q} d x \leq \sum_{i=1}^{n}\left\|F_{i}\right\|_{q}\left(\int_{\mathbf{R}^{n}}\left|\partial_{x_{i}}\left(f|f|^{q-2}\right)\right|^{q^{\prime}} d x\right)^{\frac{1}{q^{\prime}}},
$$

here $q^{\prime}$ is the conjugate exponent defined by $1 / q+1 / q^{\prime}=1$. Notice that supp $\hat{f}_{i} \subset$ $\left\{\xi \in \mathbf{R}^{n} ;\left|\xi_{i}\right| \sim 2^{j}\right\}$, thus supp $\hat{F}_{i} \subset\left\{\xi \in \mathbf{R}^{n} ;\left|\xi_{i}\right| \sim 2^{j}\right\}$, then by Lemma 2.1, we deduce that

$$
\left\|F_{i}\right\|_{q} \leq C 2^{-j}\left\|\partial_{x_{i}} F_{i}\right\|_{q}=C 2^{-j}\left\|f_{i}\right\|_{q} \leq C 2^{-j}\|f\|_{q}
$$

On the other hand, we have

$$
\left|\partial_{x_{i}}\left(f|f|^{q-2}\right)\right| \leq(q-1)|\nabla f||f|^{q-2} .
$$

Putting together our estimates, we conclude that

$$
\begin{aligned}
\int_{\mathbf{R}^{n}}|f|^{q} d x & \leq C 2^{-j}\|f\|_{q} \sum_{i=1}^{n}\left(\int_{\mathbf{R}^{n}}\left|\partial_{x_{i}}\left(f|f|^{q-2}\right)\right|^{q^{\prime}} d x\right)^{\frac{1}{q^{\prime}}} \\
& \leq C 2^{-j}\|f\|_{q}\left(\int_{\mathbf{R}^{n}}|\nabla f|^{q^{\prime}}|f|^{(q-2) q^{\prime}} \mathrm{d} x\right)^{\frac{1}{q^{\prime}}} \\
& \leq C 2^{-j}\|f\|_{q}\left(\int_{\mathbf{R}^{n}}|\nabla f|^{2}|f|^{q-2} \mathrm{~d} x\right)^{\frac{1}{2}}\left(\int_{\mathbf{R}^{n}}|f|^{q} \mathrm{~d} x\right)^{\frac{1}{q^{\prime}}-\frac{1}{2}}
\end{aligned}
$$

which implies (2.5).

Lemma 2.3 (Commutator estimate). Let $k \in \mathbb{Z}, p, q, r$ satisfy $1 \leq p, q, r \leq$ $\infty, \frac{1}{p}=\frac{1}{q}+\frac{1}{r}$. Then for any $f, g \in \mathcal{S}\left(\mathbf{R}^{n}\right)$, we have

$$
\left\|P_{k}(f \nabla g)-f P_{k}(\nabla g)\right\|_{p} \leq C\|\nabla f\|_{q}\|g\|_{r},
$$

where $C$ is a constant independent of $f$ and $g$.

Proof. First we have

$$
P_{k}\left(f \partial_{x_{i}} g\right)-f P_{k}\left(\partial_{x_{i}} g\right)=\int_{\mathbf{R}^{n}} 2^{k n} \psi\left(2^{k} y\right)(f(x-y)-f(x)) \partial_{y_{i}} g(x-y) d y,
$$

then by integration by parts, we get

$$
\begin{aligned}
& P_{k}\left(f \partial_{x_{i}} g\right)-f P_{k}\left(\partial_{x_{i}} g\right) \\
& =\int_{\mathbf{R}^{n}} 2^{(n+1) k} \partial_{y_{i}} \psi\left(2^{k} y\right)(f(x-y)-f(x)) g(x-y) d y \\
& \quad-\int_{\mathbf{R}^{n}} 2^{k n} \psi\left(2^{k} y\right) \partial_{y_{i}} f(x-y) g(x-y) d y .
\end{aligned}
$$


By the Minkowski inequality and the Hölder inequality, we can estimate the second term on the right hand side of (2.7) as follows:

$$
\left\|\int_{\mathbf{R}^{n}} 2^{k n} \psi\left(2^{k} y\right) \partial_{y_{i}} f(x-y) g(x-y) d y\right\|_{p} \leq C\|\nabla f\|_{q}\|g\|_{r} .
$$

For the first term, by the fundamental theorem of calculus, we can rewrite it as

$$
\int_{\mathbf{R}^{n}} 2^{(n+1) k} \partial_{y_{i}} \psi\left(2^{k} y\right) \int_{0}^{1} y \cdot \nabla f(x-t y) \mathrm{d} t g(x-y) d y
$$

thus, in the same way as in $(2.8)$, we have

$$
\begin{aligned}
& \left\|\int_{\mathbf{R}^{n}} 2^{(n+1) k} \partial_{y_{i}} \psi\left(2^{k} y\right)(f(x-y)-f(x)) g(x-y) d y\right\|_{p} \\
& \leq \int_{\mathbf{R}^{n}} 2^{k n}\left|2^{k} y\left\|\partial_{y_{i}} \psi\left(2^{k} y\right) \mid \int_{0}^{1}\right\| \nabla f(x-t y) g(x-y) \|_{p} d t d y\right. \\
& \leq C\|\nabla f\|_{q}\|g\|_{r} .
\end{aligned}
$$

From (2.7)-(2.9), we get (2.6).

3. Space-time estimates in the Besov spaces. In this section we prove Theorem 1.1.

Proof of Theorem 1.1. Taking the operation $P_{k}$ on both sides of (1.1), we get

$$
\left(P_{k} u^{j}\right)_{t}-\triangle P_{k} u^{j}+P_{k}\left(u^{i} \partial_{x_{i}} u^{j}\right)+\partial_{x_{j}} P_{k} \pi=0, \quad(j=1,2, \cdots, n) .
$$

Multiplying by $\left|P_{k} u^{j}\right|^{p-2} P_{k} u^{j}$ on both sides of (3.1), then integrating with respect to $x$ over $\mathbf{R}^{n}$, we get the following equality

$$
\begin{aligned}
& \int_{\mathbf{R}^{n}}\left(P_{k} u^{j}\right)_{t}\left|P_{k} u^{j}\right|^{p-2} P_{k} u^{j} d x \\
& =\int_{\mathbf{R}^{n}} \triangle P_{k} u^{j}\left|P_{k} u^{j}\right|^{p-2} P_{k} u^{j} d x-\int_{\mathbf{R}^{n}} P_{k}\left(u^{i} \partial_{x_{i}} u^{j}\right)\left|P_{k} u^{j}\right|^{p-2} P_{k} u^{j} d x \\
& \quad-\int_{\mathbf{R}^{n}} \partial_{x_{j}} P_{k} \pi\left|P_{k} u^{j}\right|^{p-2} P_{k} u^{j} d x .
\end{aligned}
$$

In what follows, we are going to calculate the above four terms separately. Firstly, we have

$$
\int_{\mathbf{R}^{n}}\left(P_{k} u^{j}\right)_{t}\left|P_{k} u^{j}\right|^{p-2} P_{k} u^{j} d x=\frac{1}{p} \frac{d}{d t} \int_{\mathbf{R}^{n}}\left|P_{k} u^{j}\right|^{p} d x .
$$

By integration by parts, we find

$$
-\int_{\mathbf{R}^{n}} \triangle P_{k} u^{j}\left|P_{k} u^{j}\right|^{p-2} P_{k} u^{j} d x=(p-1) \int_{\mathbf{R}^{n}}\left|\nabla P_{k} u^{j}\right|^{2}\left|P_{k} u^{j}\right|^{p-2} d x,
$$

then apply Lemma 2.2 to obtain

$$
-\int_{\mathbf{R}^{n}} \triangle P_{k} u^{j}\left|P_{k} u^{j}\right|^{p-2} P_{k} u^{j} d x \geq C 2^{2 k} \int_{\mathbf{R}^{n}}\left|P_{k} u^{j}\right|^{p} d x .
$$


Using the Littlewood-Paley trichotomy decomposition with $f=u^{i}, g=\partial_{x_{i}} u^{j}$, we decompose the second term on the right hand of (3.2) as follows:

$$
\begin{aligned}
& \int_{\mathbf{R}^{n}} P_{k}\left(u^{i} \partial_{x_{i}} u^{j}\right)\left|P_{k} u^{j}\right|^{p-2} P_{k} u^{j} d x \\
& =\sum_{l<k-5} \int_{\mathbf{R}^{n}} P_{k}\left(P_{k-3 \leq-\leq k+3} u^{i} P_{l}\left(\partial_{x_{i}} u^{j}\right)\right)\left|P_{k} u^{j}\right|^{p-2} P_{k} u^{j} d x \\
& \quad+\sum_{l<k-5} \int_{\mathbf{R}^{n}} P_{k}\left(P_{l} u^{i} P_{k-3 \leq-\leq k+3}\left(\partial_{x_{i}} u^{j}\right)\right)\left|P_{k} u^{j}\right|^{p-2} P_{k} u^{j} d x \\
& \quad+\int_{\mathbf{R}^{n}} P_{k}\left(P_{k-5 \leq \cdot \leq k+5} u^{i} P_{k-5} \leq \cdot \leq k+5\right. \\
& \quad+\left.\sum_{l, l^{\prime}>k+5 ;\left|l-l^{\prime}\right| \leq 3} \int_{\mathbf{R}^{n}} P_{k}\left(P_{l} u^{i} P_{l^{\prime}}\left(\partial_{x_{i}} u^{j}\right)\right)\left|P_{k} u^{j}\right|^{p-2} P_{k}\right|^{p-2} P_{k} u^{j} d x \\
& \equiv I+I I+I I I+I V .
\end{aligned}
$$

Next we estimate each term of (3.5) separately below. By the Hölder inequality and Lemma 2.1, we obtain

$$
\begin{aligned}
|I| & \leq C \sum_{l<k-5}\left\|P_{k-3 \leq-\leq k+3} u\right\|_{p}\left\|P_{k} u\right\|_{p}^{p-1}\left\|P_{l}\left(\partial_{x_{i}} u\right)\right\|_{\infty} \\
& \leq C \sum_{l<k-5} 2^{\left(\frac{n}{r}+1\right) l}\left\|P_{l} u\right\|_{r}\left\|P_{k-3 \leq \cdot \leq k+3} u\right\|_{p}\left\|P_{k} u\right\|_{p}^{p-1} .
\end{aligned}
$$

For $I I I$, in the same way as in the proof of (3.6), we also have

$$
|I I I| \leq C \sum_{k-5 \leq l \leq k+5} 2^{\left(\frac{n}{r}+1\right) l}\left\|P_{l} u\right\|_{r}\left\|P_{k-5 \leq \cdot \leq k+5} u\right\|_{p}\left\|P_{k} u\right\|_{p}^{p-1} .
$$

Since $\nabla \cdot u=0$, we find

$$
P_{k}\left(P_{l} u^{i} P_{l^{\prime}}\left(\partial_{x_{i}} u^{j}\right)\right)=\partial_{x_{i}} P_{k}\left(P_{l} u^{i} P_{l^{\prime}} u^{j}\right),
$$

then by Lemma 2.1 and the Hölder inequality, we obtain

$$
\begin{aligned}
|I V| & \leq C 2^{k}\left\|P_{k} u\right\|_{\infty}\left\|P_{k} u\right\|_{p}^{p-2} \sum_{l, l^{\prime}>k+5 ;\left|l-l^{\prime}\right| \leq 3}\left\|P_{l} u\right\|_{p}\left\|P_{l^{\prime}} u\right\|_{p} \\
& \leq C 2^{\left(\frac{n}{r}+1\right) k}\left\|P_{k} u\right\|_{r}\left\|P_{k} u\right\|_{p}^{p-2} \sum_{l, l^{\prime}>k+5 ;\left|l-l^{\prime}\right| \leq 3}\left\|P_{l} u\right\|_{p}\left\|P_{l^{\prime}} u\right\|_{p} .
\end{aligned}
$$

We next turn to the estimate of $I I$. First, we observe

$$
\begin{aligned}
& \int_{\mathbf{R}^{n}} P_{l} u^{i} P_{k}\left(P_{k-3 \leq-\leq k+3} \partial_{x_{i}} u^{j}\right)\left|P_{k} u^{j}\right|^{p-2} P_{k} u^{j} d x \\
& =\int_{\mathbf{R}^{n}} P_{l} u^{i} P_{k}\left(\partial_{x_{i}} u^{j}\right)\left|P_{k} u^{j}\right|^{p-2} P_{k} u^{j} d x \\
& =\int_{\mathbf{R}^{n}} \partial_{x_{i}}\left(P_{l} u^{i} P_{k} u^{j}\right)\left|P_{k} u^{j}\right|^{p-2} P_{k} u^{j} d x \\
& =-(p-1) \int_{\mathbf{R}^{n}} P_{l} u^{i} P_{k}\left(\partial_{x_{i}} u^{j}\right)\left|P_{k} u^{j}\right|^{p-2} P_{k} u^{j} d x=0,
\end{aligned}
$$


thus we have

$$
\begin{aligned}
I I= & \sum_{l<k-5} \int_{\mathbf{R}^{n}}\left\{P_{k}\left(P_{l} u^{i} P_{k-3 \leq \cdot \leq k+3} \partial_{x_{i}} u^{j}\right)-\right. \\
& \left.-P_{l} u^{i} P_{k}\left(P_{k-3 \leq-\leq k+3} \partial_{x_{i}} u^{j}\right)\right\}\left|P_{k} u^{j}\right|^{p-2} P_{k} u^{j} d x .
\end{aligned}
$$

On the other hand, by Lemma 2.3 and Lemma 2.1, we get

$$
\begin{aligned}
& \left\|P_{k}\left(P_{l} u^{i} P_{k-3 \leq \cdot \leq k+3} \partial_{x_{i}} u^{j}\right)-P_{l} u^{i} P_{k}\left(P_{k-3 \leq \cdot \leq k+3} \partial_{x_{i}} u^{j}\right)\right\|_{p} \\
& \quad \leq C\left\|P_{l} \nabla u\right\|_{\infty}\left\|P_{k-3 \leq \cdot \leq k+3} u\right\|_{p} \\
& \quad \leq C 2^{\left(\frac{n}{r}+1\right) l}\left\|P_{l} u\right\|_{r}\left\|P_{k-3 \leq \cdot \leq k+3} u\right\|_{p},
\end{aligned}
$$

from which we get

$$
|I I| \leq C \sum_{l<k-5} 2^{\left(\frac{n}{r}+1\right) l}\left\|P_{l} u\right\|_{r}\left\|P_{k-3 \leq \cdot \leq k+3} u\right\|_{p}\left\|P_{k} u\right\|_{p}^{p-1} .
$$

Then by summing up (3.6)-(3.9), we have

$$
\begin{aligned}
& \int_{\mathbf{R}^{n}} P_{k}\left(u^{i} \partial_{x_{i}} u^{j}\right)\left|P_{k} u^{j}\right|^{p-2} P_{k} u^{j} d x \\
& \leq C \sum_{l<k+5} 2^{\left(\frac{n}{r}+1\right) l}\left\|P_{l} u\right\|_{r}\left\|P_{k} u\right\|_{p}^{p-2} \\
& \quad \times\left(\sum_{m=k-5}^{k+5}\left\|P_{m} u\right\|_{p}^{2}+\sum_{l, l^{\prime}>k+5 ;\left|l-l^{\prime}\right| \leq 3}\left\|P_{l} u\right\|_{p}\left\|P_{l^{\prime}} u\right\|_{p}\right) .
\end{aligned}
$$

Finally, we estimate the pressure term in (3.2). We recall the well-known representation of the pressure

$$
\pi=(-\triangle)^{-1} \partial_{x_{i}} \partial_{x_{m}}\left(u^{i} u^{m}\right)
$$

which gives

$$
\partial_{x_{j}} \pi=(-\triangle)^{-1} \partial_{x_{j}} \partial_{x_{i}} \partial_{x_{m}}\left(u^{i} u^{m}\right)=(-\triangle)^{-1} \partial_{x_{j}} \partial_{x_{m}}\left(u^{i} \partial_{x_{i}} u^{m}\right) .
$$

Then exactly as in the derivation of (3.10), we have

$$
\begin{aligned}
& \int_{\mathbf{R}^{n}} \partial_{x_{j}} P_{k} \pi\left|P_{k} u^{j}\right|^{p-2} P_{k} u^{j} d x \\
& \leq C \sum_{l<k+5} 2^{\left(\frac{n}{r}+1\right) l}\left\|P_{l} u\right\|_{r}\left\|P_{k} u\right\|_{p}^{p-2} \\
& \quad \times\left(\sum_{m=k-5}^{k+5}\left\|P_{m} u\right\|_{p}^{2}+\sum_{l, l^{\prime}>k+5 ;\left|l-l^{\prime}\right| \leq 3}\left\|P_{l} u\right\|_{p}\left\|P_{l^{\prime}} u\right\|_{p}\right) .
\end{aligned}
$$

Then by summing up (3.2)-(3.4), (3.10), and (3.11), and doing summation for $j$, we finally obtain

$$
\begin{aligned}
& \frac{d}{d t}\left\|P_{k} u\right\|_{p}^{p}+2^{2 k}\left\|P_{k} u\right\|_{p}^{p} \\
& \leq C \sum_{l<k+5} 2^{\left(\frac{n}{r}+1\right) l}\left\|P_{l} u\right\|_{r}\left\|P_{k} u\right\|_{p}^{p-2} \\
& \quad \times\left(\sum_{m=k-5}^{k+5}\left\|P_{m} u\right\|_{p}^{2}+\sum_{l, l^{\prime}>k+5 ;\left|l-l^{\prime}\right| \leq 3}\left\|P_{l} u\right\|_{p}\left\|P_{l^{\prime}} u\right\|_{p}\right) .
\end{aligned}
$$


Set

$$
G_{k}(t)=\sum_{m=k-5}^{k+5}\left\|P_{m} u(t)\right\|_{p}^{2}+\sum_{l, l^{\prime}>k+5 ;\left|l-l^{\prime}\right| \leq 3}\left\|P_{l} u(t)\right\|_{p}\left\|P_{l^{\prime}} u(t)\right\|_{p} .
$$

Then the Gronwall inequality gives

$$
\left\|P_{k} u(t)\right\|_{p}^{2} \leq\left\|P_{k} u(0)\right\|_{p}^{2} e^{-2^{2 k} t}+C \sum_{l<k+5} 2^{\left(\frac{n}{r}+1\right) l} e^{-2^{2 k} t} *\left(\left\|P_{k} u\right\|_{r} G_{k}\right),
$$

where the $\operatorname{sign} *$ denotes the convolution of functions defined on $\mathbf{R}^{+}$. We take $L^{\infty}(0, T)$ norm to obtain

$$
\begin{gathered}
\left\|P_{k} u\right\|_{L_{t}^{\infty} L_{x}^{p}}^{2} \leq\left\|P_{k} u(0)\right\|_{p}^{2}+C \sum_{l<k+5} 2^{\left(\frac{n}{r}+1\right) l}\left\|P_{l} u\right\|_{L_{t}^{q} L_{x}^{r}}\left\|G_{k}\right\|_{L_{t}^{q^{\prime}}} \\
\leq\left\|P_{k} u(0)\right\|_{p}^{2}+C 2^{\left(\frac{n}{r}+1-\gamma\right) k}\|u\|_{{\widetilde{L_{T}}}^{q} \dot{B}_{r}^{\gamma, \infty}}\left\|G_{k}\right\|_{L_{t}^{q^{\prime}}},
\end{gathered}
$$

where we have used the Hölder inequality. On the other hand, we take $L^{2}(0, T)$ norm to obtain

$$
\begin{gathered}
\left\|P_{k} u\right\|_{L_{t}^{2} L_{x}^{p}}^{2} \leq\left\|P_{k} u(0)\right\|_{p}^{2}\left\|e^{-2^{2 k} t}\right\|_{L_{t}^{1}}+ \\
+C \sum_{l<k+5} 2^{\left(\frac{n}{r}+1\right) l}\left\|e^{-2^{2 k} t}\right\|_{L_{t}^{1}}\left\|P_{l} u\right\|_{L_{t}^{q} L_{x}^{r}}\left\|G_{k}\right\|_{L_{t}^{q^{\prime}}} \\
\leq C 2^{-2 k}\left\|P_{k} u(0)\right\|_{p}^{2}+C 2^{\left(\frac{n}{r}-\gamma-1\right) k}\|u\|_{{\widetilde{L_{T}}}^{q} \dot{B}_{r}^{\gamma, \infty}}\left\|G_{k}\right\|_{L_{t}^{q^{\prime}}}
\end{gathered}
$$

where we have used the Young inequality and Hölder inequality. Multiplying by $2^{2 s k}$ on both sides of (3.13), and for the case of $\sigma \geq 1$ taking $l^{\frac{\sigma}{2}}$ quasi-norm with respect to $k$, we obtain

$$
\begin{aligned}
& \|u\|_{{\widetilde{L_{T}}}^{\infty} \dot{B}_{p}^{s, \sigma}}^{2} \leq\|u(0)\|_{\dot{B}_{p}^{s, \sigma}}^{2} \\
& \quad+C\|u\|_{{\widetilde{L_{T}}}^{q} \dot{B}_{r}^{\gamma, \infty}}\left(\sum_{k} 2^{\left(\frac{n}{r}-\gamma+1+2 s\right) \frac{k \sigma}{2}}\left\|G_{k}\right\|_{L_{t}^{q^{\prime}}}^{\frac{\sigma}{2}}\right)^{\frac{2}{\sigma}} .
\end{aligned}
$$

Multiplying by $2^{2(s+1) k}$ on both sides of (3.14), and taking $l^{\frac{\sigma}{2}}$ norm, we obtain

$$
\begin{aligned}
& \|u\|_{\widetilde{L_{T}}}^{2} \dot{B}_{p}^{s+1, \sigma} \leq C\|u(0)\|_{\dot{B}_{p}^{s, \sigma}}^{2} \\
& \quad+C\|u\|_{\widetilde{L_{T}}{ }^{q} \dot{B}_{r}^{\gamma, \infty}}\left(\sum_{k} 2^{\left(\frac{n}{r}-\gamma+1+2 s\right) \frac{k \sigma}{2}}\left\|G_{k}\right\|_{L_{t}^{q^{\prime}}}^{\frac{\sigma}{2}}\right)^{\frac{2}{\sigma}} .
\end{aligned}
$$

Next we will prove the following inequality

$$
\left(\sum_{k} 2^{\left(\frac{n}{r}-\gamma+1+2 s\right) \frac{k \sigma}{2}}\left\|G_{k}\right\|_{L_{t}^{q^{\prime}}}^{\frac{\sigma}{2}}\right)^{\frac{2}{\sigma}} \leq C\|u\|_{\widetilde{L}_{T} \infty}^{\frac{2}{q}} \dot{B}_{p}^{s, \sigma}\|u\|_{\widetilde{L}_{T}^{2}}^{\frac{2}{q^{\prime}}} \dot{B}_{p}^{s+1, \sigma} .
$$

Notice that

$$
G_{k}(t) \leq C \sum_{l \geq k-5}\left\|P_{l} u(t)\right\|_{p}^{2}
$$


If we let $\theta=\frac{n}{r}-\gamma+1+2 s(\theta>0$, by assumptions $)$, then we have

$$
\begin{aligned}
& \left(\sum_{k} 2^{\theta \frac{k \sigma}{2}}\left\|G_{k}\right\|_{L_{t}^{q^{\prime}}}^{\frac{\sigma}{2}}\right)^{\frac{2}{\sigma}} \\
& \leq C\left(\sum_{k} 2^{\theta \frac{k \sigma}{2}}\left(\sum_{l \geq k-5}\left\|P_{l} u\right\|_{L_{t}^{\infty} L_{x}^{p}}^{\frac{2}{q}}\left\|P_{l} u\right\|_{L_{t}^{2} L_{x}^{p}}^{\frac{2}{q^{\prime}}}\right)^{\frac{\sigma}{2}}\right)^{\frac{2}{\sigma}} \\
& =C\left(\sum_{k} 2^{\theta \frac{k \sigma}{2}}\left(\sum_{l \geq-5}\left\|P_{l+k} u\right\|_{L_{t}^{\infty} L_{x}^{p}}^{\frac{2}{q}}\left\|P_{l+k} u\right\|_{L_{t}^{2} L_{x}^{p}}^{\frac{2}{q^{p}}}\right)^{\frac{\sigma}{2}}\right)^{\frac{2}{\sigma}}
\end{aligned}
$$

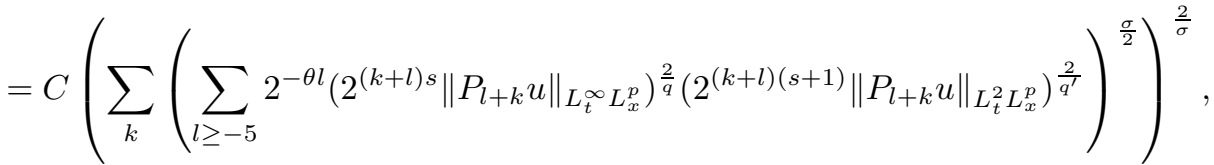

where we have used the fact $(\theta-2 s) q^{\prime}=2$ in the last equality. If $\sigma \geq 2$, we use the Minkowski inequality to obtain

$$
\begin{aligned}
& \left(\sum_{k} 2^{\theta \frac{k \sigma}{2}}\left\|G_{k}\right\|_{L_{t}^{q^{\prime}}}^{\frac{\sigma}{2}}\right)^{\frac{2}{\sigma}} \\
& \leq C \sum_{l \geq-5} 2^{-\theta l}\left(\sum_{k}\left(2^{k s \sigma}\left\|P_{k} u\right\|_{L_{t}^{\infty} L_{x}^{p}}^{\sigma}\right)^{\frac{1}{q}}\left(2^{(k(s+1) \sigma}\left\|P_{k} u\right\|_{L_{t}^{2} L_{x}^{p}}^{\sigma}\right)^{\frac{1}{q^{\prime}}}\right)^{\frac{2}{\sigma}} \\
& \leq C\|u\|_{\widetilde{L}_{T}}^{\frac{2}{q}} \dot{B}_{p}^{s, \sigma}\|u\|_{\widetilde{L}_{T}}^{\frac{2}{q^{\prime}}} \dot{B}_{p}^{s+1, \sigma} .
\end{aligned}
$$

where we have used the Hölder inequality for series in the last line. On the other hand, for $\sigma \leq 2$, we use the embedding $l^{\frac{\sigma}{2}} \hookrightarrow l^{1}$ to obtain

$$
\begin{aligned}
& \left(\sum_{k} 2^{\theta \frac{k \sigma}{2}}\left\|G_{k}\right\|_{L_{t}^{q^{\prime}}}^{\frac{\sigma}{2}}\right)^{\frac{2}{\sigma}} \\
& \leq\left(\sum_{k} 2^{\theta \frac{k \sigma}{2}} \sum_{l \geq k-5}\left\|P_{l} u\right\|_{L_{t}^{\infty} L_{x}^{p}}^{\frac{\sigma}{q}}\left\|P_{l} u\right\|_{L_{t}^{2} L_{x}^{p}}^{\frac{\sigma}{q^{\prime}}}\right)^{\frac{2}{\sigma}} \\
& \leq C\left(\sum_{l \geq-5} 2^{-\theta \sigma l / 2} \sum_{k}\left(2^{k s \sigma}\left\|P_{k} u\right\|_{L_{t}^{\infty} L_{x}^{p}}^{\sigma}\right)^{\frac{1}{q}}\left(2^{(k(s+1) \sigma}\left\|P_{k} u\right\|_{L_{t}^{2} L_{x}^{p}}^{\sigma}\right)^{\frac{1}{q^{\prime}}}\right)^{\frac{2}{\sigma}} \\
& \leq C\|u\|_{\widetilde{L}_{T}}^{\frac{2}{q}} \dot{B}_{p}^{s, \sigma}\|u\|_{\widetilde{L}_{T}^{2}}^{\frac{2}{q_{B}}} \dot{B}_{p}^{s+1, \sigma} .
\end{aligned}
$$

Putting together the above estimates, we obtain the inequality (3.17). Finally by summing up (3.15), (3.16), and (3.17), we get

$$
\begin{aligned}
& \|u\|_{\widetilde{L_{T}}}^{2} \dot{B}_{p}^{s, \sigma}+\|u\|_{{\widetilde{L_{T}}}^{2} \dot{B}_{p}^{s+1, \sigma}}^{2} \\
& \leq C\|u(0)\|_{\dot{B}_{p}^{s, \sigma}}^{2}+C\|u\|_{\widetilde{L_{T}}{ }^{q} \dot{B}_{r}^{\gamma, \infty}}\|u\|_{\widetilde{L_{T}}}^{\frac{2}{q}} \dot{B}_{p}^{s, \sigma}\|u\|_{{\widetilde{L_{T}}}^{2} \dot{B}_{p}^{s+1, \sigma}}^{\frac{2}{q^{\prime}}} .
\end{aligned}
$$


Then apply the Young inequality to obtain

$$
\|u\|_{\widetilde{L}_{T}^{\infty} \dot{B}_{p}^{s, \sigma}}^{2}+\|u\|_{{\widetilde{L_{T}}}^{2} \dot{B}_{p}^{s+1, \sigma}}^{2} \leq C\|u(0)\|_{\dot{B}_{p}^{s, \sigma}}^{2}+C\|u\|_{{\widetilde{L_{T}}}^{q} \dot{B}_{r}^{\gamma}}^{q}\|u\|_{{\widetilde{L_{T}}}^{\infty} \dot{B}_{p}^{s, \sigma}}^{2} .
$$

This completes the proof of Theorem 1.1.

4. Proof of Theorem 1.2 and Theorem 1.3. Let us firstly prove Theorem 1.2 .

Proof of Theorem 1.2. Take $\varepsilon_{0}>0$ such that $C \varepsilon_{0} \leq \frac{1}{4}$, where $C$ is the constant in Theorem 1.1. Then we choose $\varepsilon>0$ such that

$$
\sup _{j} 2^{j \gamma q} \int_{T-\varepsilon}^{T}\left\|P_{j} u(t)\right\|_{r}^{q} d t \leq 2 \varepsilon_{0} .
$$

Thus by Theorem 1.4 with $p=\sigma=2$, we have

$$
\|u\|_{L^{\infty}\left([T-\varepsilon, T) ; H^{s}\right)} \leq 2 C\|u(T-\varepsilon)\|_{H^{s}} .
$$

Hence by a standard argument of continuation of local solutions, the solution $u(t)$ can be continued after $t=T$.

To complete the proof of Theorem 1.3, we will need the following two propositions.

Proposition 4.1. [16] Let $p>n$. Suppose $u_{0} \in L^{p}\left(\mathbf{R}^{n}\right)$ satisfying $\nabla \cdot u_{0}=0$. Then there is $T_{0}>0$ and a unique mild solution of (NS) on $\left[0, T_{0}\right)$ such that $u \in$ $C\left(\left[0, T_{0}\right) ; L^{p}\left(\mathbf{R}^{n}\right)\right)$; Furthermore, let $\left[0, T_{*}\right)$ be the maximal interval such that $u$ solves the Navier-Stokes equations in $C\left(\left[0, T_{*}\right) ; L^{p}\left(\mathbf{R}^{n}\right)\right)$. Then

$$
\|u(t)\|_{p} \geq \frac{C}{\left(T_{*}-t\right)^{\frac{p-n}{2 p}}}, \quad \forall 0 \leq t<T_{\star},
$$

with constant $C$ independent of $T_{*}$ and $p$.

Proposition 4.2. [16] Let $u, v$ be two weak solutions of $(N S)$ on $(0, T)$. Suppose that $v \in L^{r}\left(0, T ; L^{p}\left(\mathbf{R}^{n}\right)\right)$, with $\frac{2}{r}+\frac{n}{p}=1, p>n$. Then u agrees with $v$ on $(0, T) \times \mathbf{R}^{n}$.

Proof of Theorem 1.3. Since $u_{0} \in H^{s}, s>\frac{n-2}{2}$, by the Sobolev imbedding theorem, there exists $p>n$ such that $u_{0} \in L^{p}\left(\mathbf{R}^{n}\right)$. Then by Proposition 4.1, there is $T_{0}>0$ and a unique mild solution of (NS) on $\left[0, T_{0}\right)$ such that $v \in C\left(\left[0, T_{0}\right) ; L^{p}\left(\mathbf{R}^{n}\right)\right)$. Let $\left[0, T_{*}\right)$ be the maximal existence interval of $v$. Obviously $v$ are both a weak solution and a regular solution. Thus by Proposition 4.2, $u$ agrees with $v$ on $\left(0, T_{*}\right) \times \mathbf{R}^{n}$. Now, if $T_{*}=T$, the proof is complete; Otherwise, by Proposition 4.1 again, we have

$$
\|u(t)\|_{p} \geq \frac{C}{\left(T_{*}-t\right)^{\frac{p-n}{2 p}}}, \quad \forall t \in\left[0, T_{*}\right) .
$$

On the other hand, by our assumptions, for $T_{*} \in(0, T)$, we can choose $\varepsilon_{0}, \varepsilon$ such that

$$
\sup _{j} 2^{j \gamma q} \int_{T_{*}-\varepsilon}^{T_{*}}\left\|P_{j} u(t)\right\|_{r}^{q} d t \leq 2 \varepsilon_{0} \leq \frac{1}{2 C}
$$

where $C$ is the constant in Theorem 1.1. Thus by Theorem 1.1 and the Sobolev embedding theorem, we have

$$
\sup _{T_{*}-\varepsilon \leq t<T_{*}}\|u(t)\|_{p} \leq C \sup _{T_{*}-\varepsilon \leq t<T_{*}}\|u(t)\|_{H^{s}} \leq C\left\|u\left(T_{*}-\varepsilon\right)\right\|_{H^{s}} \leq C,
$$


which contradicts with (4.2). This completes the proof of Theorem 1.3.

Acknowledgement. We would like to thank the referees for making helpful comments on improving presentation of this paper. The first author is supported by Tianyuan Foundation of Mathematics(10526010). The second author is supported by National Natural Science Foundation of China(10601002).

\section{REFERENCES}

[1] Beirão da Veiga H., A new regularity class for the Navier-Stokes equations in $\mathbf{R}^{n}$, Chinese Ann. of Math., 16B (1995), pp. 407-412.

[2] Bergh J. and Löfstrom J., Interpolation spaces, An Introduction, Berlin New York: SpringerVerlag, 1976.

[3] Bony J.-M., Calcul symbolique et propagation des singularitiés pour les équations aux dérivées partielles non linéaires, Ann. de l'Ecole Norm. Sup., 14(1981), pp. 209-246.

[4] Caffarelli L., Kohn R., And Nirenberg L., Partial regularity of suitable weak solutions of the Navier-Stokes equations, Comm. Pure Appl. Math., 35 (1982), pp. 771-831.

[5] Cannone M., A generalization of a theorem by Kato on Navier-Stokes equations, Revista Matemática Iberoamericana, 13 (1997), pp. 515-541.

[6] Cannone M. and Planchon F., On the non stationary Navier-Stokes equations with an external force, Adv. Differential Equations, 4 (1997), pp. 697-730.

[7] Chat D., Remarks on the blow-up of the Euler equations and the related equations, Comm. Math. Phys., 245 (2004), pp. 539-550.

[8] Chemin, J.-Y. And Lerner N., Flot de champs de vecteurs non lipschitziens et êquations de Navier-Stokes, J. Differential Equations, 121 (1995), pp. 314-328.

[9] Chemin, J.-Y., Perfect incompressible fluids, Oxford Lecture Series in Mathematics and its Applications, 14. New York: The Clarendon Press, Oxford University Press, 1998. Translated from the 1995 French original by Isabelle Gallagher and Dragos Iftimie.

[10] Chen, Z. M. And Xin, Z., Homogeneity criterion of the Navier-Stokes equations in the whole spaces, J. Math. Fluid Mech., 3 (2001), pp. 152-182.

[11] Constantin P. and Feffermann C., Direction of vorticity and the problem of global regularity for the Navier-Stokes equations, Indiana Univ. Math. J., 42 (1993), pp. 775-789.

[12] Danchin D., Global existence in critical spaces for compressible Navier-Stokes equtions, Invent. Math., 141 (2000), pp. 579-614.

[13] Fabes E. B.,Jones B. F., And Riviere N. M., The initial value problem for the Navier-Stokes equations with data in $L^{p}$, Arch. Rational Mech. Anal., 45 (1972), pp. 222-240.

[14] Fujita H. And Kato T., On the Navier-Stokes initial problem 1, Arch. Rational Mech. Anal., 16 (1964), pp. 269-315.

[15] Gallagher I. and Planchon F., On infinite energy solutions to the Navier-Stokes equations: global 2D existence and 3D weak-strong uniqueness, Arch. Ration. Mech. Anal., 161 (2002), pp. 307-337.

[16] Giga Y., Solutions for semilinear parabolic equations in $L^{p}$ and regularity of weak solutions of the Navier-Stokes system, J. Differential Equations, 62 (1986), pp. 186-212.

[17] Hopf E., Ueber die Anfangswertaufgbe für die hydrodynamischen Grundgleichungen, Math. Nachr., 4 (1951), pp. 213-231.

[18] Kozono H. And Taniuchi Y., Bilinear estimates in BMO and the Navier-Stokes equations, Math. Z., 235 (2000), pp. 173-194.

[19] Kozono H., Ogawa T., and Taniuchi Y., The critical Sobolev inequalities in Besov spaces and regularity criterion to some semi-linear evolution equations, Math. Z., 242 (2002), pp. 251-278.

[20] Leray J., Sur le mouvement d'un liquids visqeux emplissant l'espace, Acta Math., 63 (1934), pp. $193-248$.

[21] Masuda K., Weak solutions of Navier-Stokes equations, Tohoku Math. J., 36 (1984), pp. 623646.

[22] Ogawa T. AND TANiuchi, The limiting uniquness criterion by vorticity to the Navier-Stokes equations, Tohoku Math. Jour., 56 (2004), pp. 65-77.

[23] Planchon F., An extension of the Beal-Kato-Majda criterion for the Euler equations, Comm. Math. Phys., 232 (2003), pp. 319-326.

[24] SERRIN J., On the interior regularity of weak solutions of the Navier-Stokes equations, Arch. Rational Mech. Anal., 9 (1962), pp. 187-195. 
[25] SERRIN J., The initial value problem for the Navier-stokes equations, In: langer R. E. (ed.) Nonlinear problems, Wisconsin: Univ. Wisconsin Press, 1963, pp. 69-98.

[26] Sohr H., Zur Reguläritatstheorie der instätionaren Gleichungen von Navier-Stokes, Math. Z., 184 (1983), pp. 359-375.

[27] Struwe M., On partial regularity results for the Navier-Stokes equations, Comm. Pure Appl. Math., 41 (1988), pp. 437-458.

[28] TAKahashi S., On interior regularity criteria for weak solutions of the Navier-Stokes equations, Manuscripta Math., 69 (1990), pp. 237-254.

[29] TAO T., Time-frequency analysis, http://www.math.ucla.edu/ tao/.

[30] Temam R., Navier-Stokes equations, Amsterdan New York Oxford: North-Holland, 1977.

[31] Triebel H., Theory of Function Spaces, Monograph in mathematics, Vol.78, Basel: Birkhauser Verlag, 1983.

[32] Vishik M., Hydrodynamics in Besov spaces, Arch. Ration. Mech. Anal., 145 (1998), pp. 197214. 